\title{
REFLEXIONES SOBRE LA INVESTIGACIÓN E INNOVACIÓN EN LOS INSTITUTOS TÉCNICOS Y TECNOLÓGICOS DEL ECUADOR.
}

\author{
Autor. \\ Rolando J. Alvarez \\ Docente titular Instituto Tecnológico \\ Illingworth
}

\author{
Coautor. \\ Lázaro R. Cruz \\ Docente titular Instituto Tecnológico \\ Illingworth
}

País Origen: Ecuador

Palabras claves: Formación, Inovación, investigación, conocimiento

Keywords: Bonding: Training , innovation , research, knowledge
Recibido: 11 de Marzo del 2016

Aceptado: 15 de Abril del 2016

\section{SUMMARY}

$T$

he present investigation speeches as an object of study process research and innovation in technical and technological institutions in the under the Ecuadorian project for construction society knowledge and the National Plan for Good Living. From the legal context governs the activity of research and technological innovation, making a conceptualization of this latter aspect and assessing their joint process academic training. Where it shows clearly the importance investigation and innovation in economic development of countries, compared pressing social problems, such as pollution, problems health, poverty and unemployment. Investigation and innovation should no longer be the exclusive prerogative of developed countries.

\section{RESUMEN}

a presente investigación aborda como objeto de estudio el proceso de investigación e innovación en las instituciones técnicas y tecnológicas en el marco del proyecto ecuatoriano para la construcción de la sociedad del conocimiento y el Plan Nacional del Buen Vivir. A partir del contexto legal que rige la actividad de investigación e innovación tecnológica, realizando una conceptualización de este último aspecto y valorando su articulación al proceso de formación académica. Donde se deja en evidencia la importancia que tiene la Investigación y la innovación en el desarrollo económico de los países, frente a problemas sociales apremiantes, como la contaminación, los problemas de salud, la pobreza y el desempleo. La investigación y la innovación deben dejar de ser una prerrogativa exclusiva de los países desarrollados. 


\section{INTRODUCCIÓN}

a investigación se constituye en uno de los ejes fundamentales que debe estar presente en las instituciones de Educación Superior, tanto en el proceso de enseñanza aprendizaje, como en el proceso de su producción científica. Este principio es reconocido a nivel constitucional en el Ecuador que en su artículo 350 establece que "El sistema de educación superior tiene como finalidad la formación académica y profesional con visión científica y humanista; la investigación científica y tecnológica; la innovación .... la construcción de soluciones para los problemas del país, en relación con los objetivos del régimen de desarrollo."

La UNESCO (2015) destacó recientemente que la investigación es un factor clave para impulsar el desarrollo sostenible y para la aceleración del crecimiento económico.

El Reglamento de Régimen Académico (RRA) vigente (2015) incluye varios artículos relacionados con la investigación. Así, el art 72, se refiere a la Investigación para el aprendizaje, que en su numeral 1, establece que "La investigación en la educación superior técnica y tecnológica se desarrollará en el campo formativo de creación, adaptación e innovación tecnológica, mediante dominio de técnicas investigativas de carácter exploratorio"

La organización de los aprendizajes en cada nivel de formación de la educación superior se sustentará en el proceso de investigación correspondiente y propenderá al desarrollo de conocimientos y actitudes para la innovación tecnológica.

Por otra parte, en el artículo 75 del Reglamento mencionado, se hace referencia a la Investigación institucional,, donde se explicita que las instituciones de educación superior, a partir de sus fortalezas o dominios académicos, deberá contar con líneas, programas y proyectos de investigación articulados en redes académicas nacionales e internacionales, guardando correspondencia con los requerimientos, prioridades y propósitos del Plan Nacional de Desarrollo, de los planes regionales y locales de desarrollo...sin prejuicio de que se respete el principio de autodeterminación para la producción del pensamiento y conocimiento, en el marco del diálogo de saberes, pensamiento universal y producción científica tecnológica global.

El informe "Índice Mundial de Innovación 2015" destaca que las políticas de innovación desempeñan una función esencial para las economías en desarrollo y las economías emergentes, en las que el fomento de la innovación está llamado a ser aspecto central de los planes y estrategias de desarrollo y una clave para hacer frente a problemas sociales apremiantes, como la contaminación, los problemas de salud, la pobreza y el desempleo.

El presente trabajo tiene como propósito reflexionar sobre el proceso de investigación e innovación en los Institutos Superiores Tecnológicos en el marco del proyecto ecuatoriano para la construcción de la sociedad del conocimiento y el Plan Nacional del Buen Vivir, a partir del contexto legal que rige la actividad de investigación e innovación tecnológica, realizando una conceptualización de este último aspecto y valorando su articulación al proceso de formación académica. Lo anterior se desarrolla tomando como referencia la experiencia de trabajo de los autores en el Instituto Tecnológico Superior Almirante Illingworth.

\section{DESARROLLO}

El Plan Nacional del Buen Vivir establece de modo detallado los objetivos a cumplimentar para la construcción de la sociedad del conocimiento, meta suprema del modelo de desarrollo ecuatoriano. Esto demanda un proceso de redimensionamiento del sistema de educación, en particular de los Institutos de Educación Superior (IES), Ilamados a ser centros formadores de los actores que se convierten en agentes de cambio capaces de implementar transformaciones e innovaciones en los procesos asociados a la producción de bienes y prestación de servicios. Solo mediante la creación del conocimiento se generará la capacidad necesaria para la transformación de la sociedad.

En este contexto, resulta de vital importancia para las IES estructurar el proceso de formación desde una perspectiva dinámica que permita el desarrollo de las capacidades investigativas articuladas a la solucion de problematicas y necesidades existentes en el entorno en el que se desenvuelve el estudiante, quien dejará de ser observador pasivo de las barreras, limitaciones y obstáculos para convertirse en actor investigador de dichas problemáticas, capaz de ofrecer soluciones innovadoras mediante un proceso de investigación - acción.

Al caracterizar la investigación en los institutos técnicos y tecnológicos, es necesario distinguir tres grandes áreas de aplicación, interrelacionadas: una primera que se refiere a la investigación para el aprendizaje, que otros autores denominan como Formación Investigativa, y algunos otros como investigación formativa y que bajo cualquier denominación se refiere al proceso de obtención y aplicación de conocimientos por parte de los estudiantes que va preparándolos para el desarrollo de competencias, habilidades y destrezas para la investigación mediante elcumplimiento d elos objetivos de formacion en investigacion a lo largo de los diferentes niveles de organización curricular. 
La segunda área tiene que ver con la investigación institucional o investigación generativa, que no sólo está relacionada con la producción científica de los profesores, sino que debe articular mediante sus líneas de investigación, programas y proyectos con la investigación para el aprendizaje en los que se insertan estudiantes ayudantes de investigación. Los proyectos desarrollados dentro de esta área incluye aquellos en los que participa el Instituto como integrante de redes de investigación, sean estas nacionales o internacionales.

El área restante corresponde a la innovación tecnológica, área sumamente importante y que no presenta un desarrollo adecuado en los momentos actuales en los Institutos.

Es justamente esta área en la que se producen nuevos productos tecnológicos y, como tal, se convierte en catalizador de la competitividad, el crecimiento y fuente de generación de empleo, teniendo como premisa las necesidades y problemáticas contenidas en el Plan Nacional de Desarrollo, los Planes de desarrollo locales, la contribución a empresas, tanto públicas como privadas, y en general a actividades productivas y de servicio.

En la figura No 1, se visualiza la estructura general que a nuestro criterio debe tener la Investigación e innovación en el Instituto.

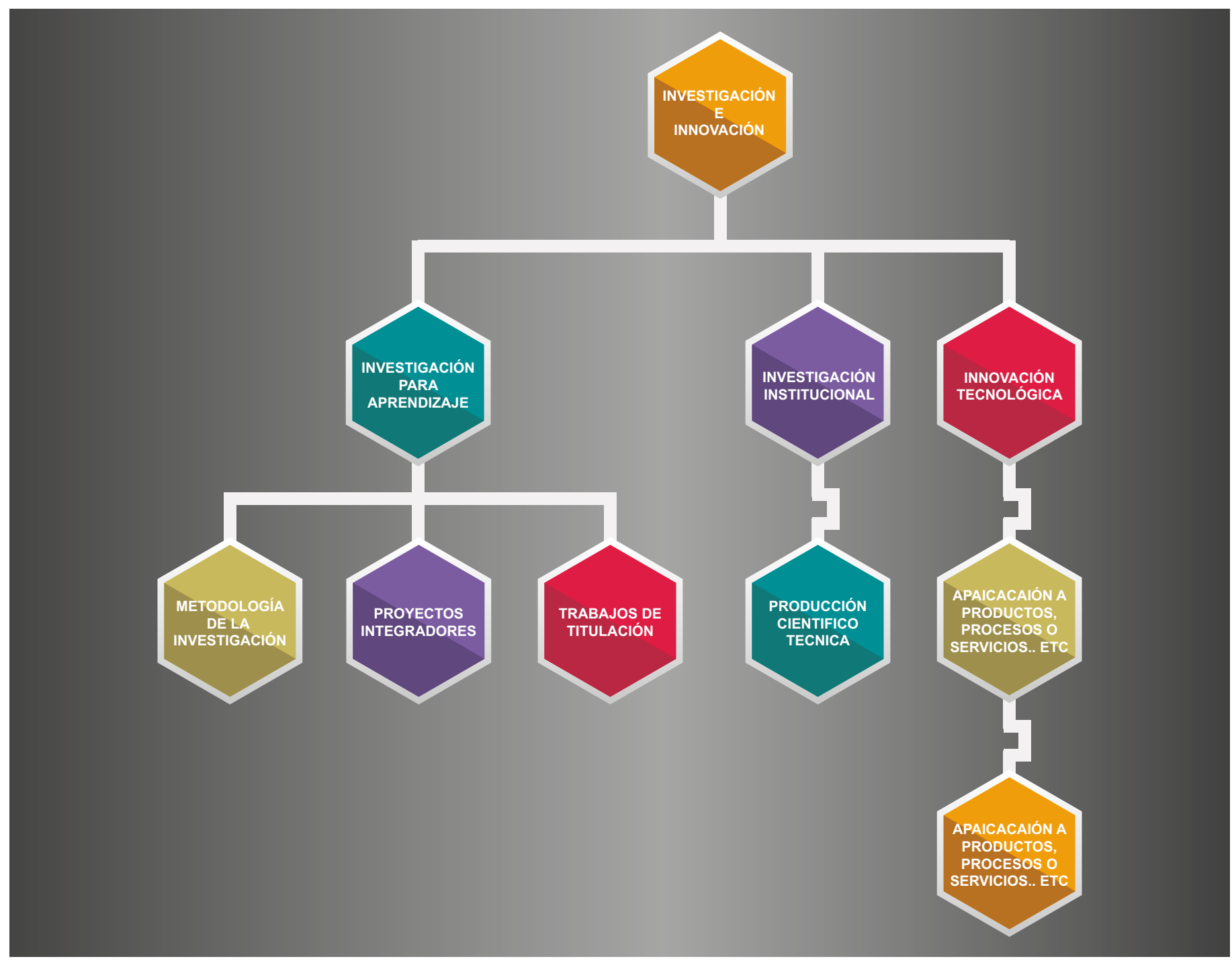

En este contexto, se puede profundizar lo relacionado con la investigación para el aprendizaje y tratar de conceptualizar lo establecido en el artículo 72, en cuanto a sus aspectos medulares, es decir, lo relacionado en el campo formativo de creación, adaptación e innovación tecnológica, mediante el desarrollo de técnicas investigativas de carácter exploratorio.

Comentemos algunos aspectos que consideramos importantes con relación a la innovación tecnológica, y su rol en los Institutos. 
Particular relevancia y prioridad se concede al proceso de transformación de una idea innovadora en nuevos productos y servicios. Varias pueden ser sus acepciones. La innovación tecnológica, no es más que la creación de valor a través de la introducción de algo nuevo en la empresa o en el mercado.

Formalizando el concepto, según Sherman Gee (1981) "es el proceso en el cual, a partir de una idea, invención o reconocimiento de necesidad se desarrolla un producto, técnica o servicio útil hasta que sea aceptado comercialmente".

Por otra parte en el Manual de Oslo (2005) se define como la introducción de un nuevo (o significativamente mejorado) producto (bien o servicio), de un proceso, de un nuevo método de comercialización o de un nuevo método organizativo en las prácticas internas de la empresa, la organización del lugar de trabajo o las relaciones exteriores.

Resulta generalmente aceptado el hecho de que el estado de la ciencia, la tecnología y la innovación es una medida del grado de desarrollo de los países No obstante, es de notar que, en cuanto a la innovación, cada vez resulta más evidente que el grado de desarrollo de este importante concepto en un país está mucho más asociado al diseño e implementación de políticas efectivas de gestión y aplicación de la innovación que a la posición que ocupa la economía de dicho país en el contexto mundial.

La innovación debe dejar de ser una prerrogativa exclusiva de los países desarrollados; debe desempeñar una función fundamental como motor del crecimiento económico y la prosperidad de la economía de los países emergentes que abren espacio para la democratización del conocimiento.

Consecuentemente, para satisfacer estos requerimientos, los Institutos Tecnológicos debemos tener la voluntad y el direccionamiento adecuado, para establecer políticas que permitan el desarrollo de ideas y proyectos innovadores entre nuestros profesores y estudiantes. Será necesario implementar estrategias metodológicas motivadoras que tenga al estudiante en su centro y rescaten los métodos colaborativos para el logro de los objetivos planteados, haciendo uso de recursos tecnológicos a la altura de las nuevas necesidades, lo cual, a su vez, demanda de un equipo de docentes competente, con habilidades amplias para desarrollar los contenidos de aprendizaje de las materias haciendo uso de las herramientas más modernas como son las Tecnologías de la Información y las Comunicaciones (TIC's).

Mención aparte merece el asunto de los límites dentro de los cuales se desarrollará la actividad de investigación y la innovación como tal. Si bien es cierto que existen programas de estudio y contenidos a impartir, adoptar metodologías que hagan prevalecer la rigidez de los programas y contenidos y mínima autonomía de trabajo, tanto de docentes como de los propios estudiantes, limitará considerablemente la capacidad de innovar. La innovación demanda flexibilidad de programas que permita adecuarlos a los escenarios cambiantes y autonomía para la utilización de los recursos tecnológicos al alcance de los estudiantes.

Conviene señalar que docentes y estudiantes participantes del proceso de investigación - acción se convierten en creadores de valor para la IES a través de los nuevos recursos tecnológicos resultante de la innovación, cuya autoría y titularidad debidamente protegidos constituyen nuevos activos de dicha IES.

Para tener una idea de la situación actual y el camino por recorrer, conviene revisar el estado de la innovación en el país y su posición relativa en el mundo, para lo cual se tomará como base el informe donde anualmente se dan los resultados del Índice Mundial de Innovación, que se ha establecido como fuente de referencia destacada en materia de innovación, y como valioso instrumento de análisis comparativo para los encargados de formular políticas en ese campo.

Particular relevancia y prioridad se concede al proceso de transformación de una idea innovadora en nuevos productos y servicios. Varias pueden ser sus acepciones. La innovación tecnológica, no es más que la creación de valor a través de la introducción de algo nuevo en la empresa o en el mercado.

Formalizando el concepto, según Sherman Gee (1981) "es el proceso en el cual, a partir de una idea, invención o reconocimiento de necesidad se desarrolla un producto, técnica o servicio útil hasta que sea aceptado comercialmente".

Por otra parte en el Manual de Oslo (2005) se define como la introducción de un nuevo (o significativamente mejorado) producto (bien o servicio), de un proceso, de un nuevo método de comercialización o de un nuevo método organizativo en las prácticas internas de la empresa, la organización del lugar de trabajo o las relaciones exteriores. 
Resulta generalmente aceptado el hecho de que el estado de la ciencia, la tecnología y la innovación es una medida del grado de desarrollo de los países No obstante, es de notar que, en cuanto a la innovación, cada vez resulta más evidente que el grado de desarrollo de este importante concepto en un país está mucho más asociado al diseño e implementación de políticas efectivas de gestión y aplicación de la innovación que a la posición que ocupa la economía de dicho país en el contexto mundial.

La innovación debe dejar de ser una prerrogativa exclusiva de los países desarrollados; debe desempeñar una función fundamental como motor del crecimiento económico y la prosperidad de la economía de los países emergentes que abren espacio para la democratización del conocimiento.

Consecuentemente, para satisfacer estos requerimientos, los Institutos Tecnológicos debemos tener la voluntad y el direccionamiento adecuado, para establecer políticas que permitan el desarrollo de ideas y proyectos innovadores entre nuestros profesores y estudiantes. Será necesario implementar estrategias metodológicas motivadoras que tenga al estudiante en su centro y rescaten los métodos colaborativos para el logro de los objetivos planteados, haciendo uso de recursos tecnológicos a la altura de las nuevas necesidades, lo cual, a su vez, demanda de un equipo de docentes competente, con habilidades amplias para desarrollar los contenidos de aprendizaje de las materias haciendo uso de las herramientas más modernas como son las Tecnologías de la Información y las Comunicaciones (TIC's).

Mención aparte merece el asunto de los límites dentro de los cuales se desarrollará la actividad de investigación y la innovación como tal. Si bien es cierto que existen programas de estudio y contenidos a impartir, adoptar metodologías que hagan prevalecer la rigidez de los programas y contenidos y mínima autonomía de trabajo, tanto de docentes como de los propios estudiantes, limitará considerablemente la capacidad de innovar. La innovación demanda flexibilidad de programas que permita adecuarlos a los escenarios cambiantes y autonomía para la utilización de los recursos tecnológicos al alcance de los estudiantes.

Conviene señalar que docentes y estudiantes participantes del proceso de investigación - acción se convierten en creadores de valor para la IES a través de los nuevos recursos tecnológicos resultante de la innovación, cuya autoría y titularidad debidamente protegidos constituyen nuevos activos de dicha IES.

Para tener una idea de la situación actual y el camino por recorrer, conviene revisar el estado de la innovación en el país y su posición relativa en el mundo, para lo cual se tomará como base el informe donde anualmente se dan los resultados del Índice Mundial de Innovación, que se ha establecido como fuente de referencia destacada en materia de innovación, y como valioso instrumento de análisis comparativo para los encargados de formular políticas en ese campo.

El informe, publicado conjuntamente por la Organización Mundial de Propiedad Intelectual (WIPO), la Universidad Cornell y la Escuela de Negocios INSEAD, establece una clasificación anual de las capacidades y los resultados de la innovación en las economías de todo el mundo.

El Índice trata de mejorar la forma de medir y comprender la innovación. En él se reconoce la necesidad de disponer de una visión amplia de la innovación, aplicable por igual a las economías desarrolladas y emergentes, y se exponen indicadores que van más allá de los métodos tradicionales empleados para medir la innovación, como el nivel de investigación y desarrollo.

Del Índice Mundial de la Innovación 2015 se desprende que una de las herramientas del éxito reside en contar con un plan y políticas de innovación que tenga objetivos claros y esté alineado con el Plan Nacional de desarrollo y las Agendas de los Gobiernos Autónomos Descentralizados (GAD). Esta articulación se pone a prueba en tiempos de dificultades económicas; cuando los recursos financieros escasean, los recursos cognitivos y la capacidad de adaptación e innovación se convierten en ventajas competitivas que pueden materializarse a modo de valor agregado a los procesos o nuevos productos.

Los resultados del informe del mencionado índice muestran que América Latina y el Caribe es una región cuyo potencial de innovación está aumentando pero sigue prácticamente sin aprovecharse. Los países suramericanos mejor representados entre los 141 incluidos en el informe son: Brasil (70), Argentina (72), y México (57) que sobresalen por ser economías con resultados por encima de la media obtenida en la región. Los excelentes resultados que obtienen regularmente Chile (42), Costa Rica (51) y Colombia (67), a nivel regional y en comparación con países que tienen un grado similar de desarrollo económico, son dignos de mención, como también lo es el nuevo papel del Perú (71) y el Uruguay (68). Ecuador se ubica en el puesto 119 de los 141 países que se incluyen en el cálculo del índice, superando solamente a Venezuela.

La época de las economías sustentadas en recursos finitos va llegando a su fin; la solución para el crecimiento económico sostenido radica en invertir en investigación e innovación. Corresponde a los Institutos Técnicos y Tec- 
nológicos implementar las estrategias necesarias para garantizar las capacidades investigativas e innovadoras a lo largo del proceso de formación académica de profesionales para el cumplimiento de esta misión social

\section{CONCLUSIONES}

Se evidencia la importancia que tiene la Investigación y la innovación en el desarrollo económico de los países, frente a problemas sociales apremiantes, como la contaminación, los problemas de salud, la pobreza y el desempleo. La investigación y la innovación deben dejar de ser una prerrogativa exclusiva de los países desarrollados

Los Institutos entonces, además de apuntar a la investigación para el aprendizaje y la investigación institucional, tenemos una gran oportunidad de dirigir los esfuerzos a desarrollar proyectos de innovación nuestros estudiantes y profesores, vinculados al Plan Nacional de Desarrollo, a los Programas de desarrollo local, y a la satisfacción de necesidades de empresas y a la población en general.

Se deben crear políticas y estructuras, que permitan tanto el desarrollo de la investigación y la innovación en nuestros Institutos, a la vez que permitan constituirnos en Redes temáticas de innovación, a partir de la creación de Centros de Gestión e Innovación Tecnológica, que abarquen todas las áreas de conocimientos.

\section{BIBLIOGRAFÍA}

UNESCO Science report: towards 2030. UNESCO 2015

CES: Reglamento de Régimen Académico codificado. Septiembre 2015

Gee Sherman, Technology transfer, Innovation\&International Competitiveness/Sherman Gee. Nueva York. Whiley\&Sons.1981

OCDE/Euroestat, Manual de Oslo: Guía para la recogida e interpretación de datos sobre innovación.2005 Soumitra Dutta, et al. The global innovation Index 2015: Effective Innovation Polices for development. 